\title{
Estudio de 100 Casos de Hemorragia Uterina con Legrado-Biopsia
}

\author{
Doctores: Germán Jordán y Enrique Darnalt
}

Contribución del Departamento de Ginecología del Instituto Nacional de Cancerologia a la CONVENCION DE OBSTETRICIA Y GINECOLOGIA.

1.-Generalidades clínicas sobre Adenocarcinoma Uterino e Hiperplasia endometrial.

El cáncer del cuerpo uterino se presenta con mavor frecuencia en la sexta década de la vida y en relación con el cáncer del cuello uterino en una proporción de un caso por ocho respectivamente.

Su evolución y sintomatologia mucho más benignas y lentas que las del cáncer del cuello, hacen más fácil su tratamiento y favorable su pronóstico. A esto «e agrega que su síntoma principal o sea la hemorragia uterina se manifiesta ránidamente, la paciente observa facilmente éste sintoma, ya sea por alteraciones he su ciclo menstrual o por aparicion de hemorragia patológica durante la me- pausia, hechos que la hacen acudir a fin de consultar dicho trastorno.

Teniendo en cuenta que el carcinoma del útero se presenta generalmente (n) las mujeres que están entre la cuarta y séptima década de su vida, el clínico debe tener en mente la posibilidad de esta entidad en toda mujer que presente hemorragia patológica. El hallazgo de un aumento del tamaño de la matriz, acompañado de hemorragia en paciente pre o menopáusica requiere un estudio cuidadoso complementado con exámenes anatomopatológicos con el fin de descartar un posible carcinoma, ya que estos mismos signos clínicos los presentan otras en:idades de tipo benigno, especialmente la hiperplasia endometrial simple o asoci.da a una fibrosis uterina o a tumores ováricos de las células de la granulosa o cales.

Teniendo en cueñta la alta incidencia de la Hiperplasia endometrial como cuusa de hemorragia uterina, es necesario aclarar algunos conceptos sobre élla. Se ha establecido claramente en observaciones y experimentos en animales que su 
causa inmediata es una estimulación excesiva del endometrio por los estrógenos acompañada de hiperactividad de la hormona foliculo-estimulante de la hipofisis. A menudo se acompaña de persistencia quística del foliculo como lo afroto Schroeder y de ausencia de la acción de la hormona luteínica y de modificaciones secretorias del endometrio. Debe distinguirse del endometrio estrógeno perpersistente causa de modificaciones menstruales con ciclos frecuentes anovulatorios o monofásicos y con reacciones hiperplásicas que se atribuyen únicamente a la falta de acción de la progesterona. Una de las más interesantes obser vaciones de los últimos años es el hallazgo de típicas modificaciones de hiperplasia en el endometrio de enfremas después de la menopausia, ya que se consideraba propia esta entidad del período reproductivo. Generalmente el tiempo de menopausia es corto pero no es raro que sea de uno o dos años y Novak trae observaciones de cinco años.

En muchas ocasiones la causa se encuentra en tumores ováricos funcionantes (verbigracia de células de la granulosa) a veces muy pequeños, pero otras no existe tumor ovárico alguno y se encuentran ovarios atróficos incapaces de producción hormonal. Se acepta generalmente que la corteza suprarrenal produce estrógenos probablemente derivados de los esteroides orgánicos propios de esta glándula. Otras escuelas creen que la hormona folículo-estimulante puede obrar directamente sobre el endometrio. Se ha discutido la relación que tiene la hiperplasia endometrial con el desarrollo posterior de carcinoma de endometrio sin que se haya llegado a conclusión alguna, aunque los autores se inclinan a creer que predispone y precede a la aparición de éste. Después de haber cureteado centenares de mujeres por presentar hiperplasia endometrial, no se han observado síntomas clínicos o histológicos de adenocarcinoma posteriormente. Generalmente se acepta que lo que sucede es la asociación de las dos lesiones. porque según las es. tadísticas de Novak y Yui se confirmaron en 104 casos estudiados 25, en los cuales coincidian carcinoma e hiperplasia. Roberto Mever aporta cinco casos con la misma asociación. Con estas consideraciones se debe tener siempre en cuenta que toda hemorragia genital por hiperplasia endometrial en mujeres pre y menopausicas debe ser estudiada por medio del legrado biópsia para descartar la presencia del adenocarcinoma o la asociación de las dos entidades.

Se ha observado que las lesiones crónicas de la célula hepatica que entre otras de sus muchas funciones metaboliza los esteroides, puede provocar una hiperplasia endometrial por aumento de las hormonas estrogenicas circulantes, que no son destruídas como sucede con hígados funcionalmente normales.

Es importante advertir que la hiperplasia endometrial no es de por sí causante de hemorragia uterina y sólo significa una excitación y una respuesta anormal del endometrio, pero que frecuentemente el epitelio hiperplasico sufre ne crobiosis por áreas con cambios degenerativos, infiltración de células redondas y trombosis de los bazos, lo que causa hemorragia funcional a veces continua, por presentarse la necrobiosis sólo en ciertas zonas por un mecanismo semejante al que causa la menstruación normal. 
Histológicamente el carcinoma del cuerpo puede clasificarse en adenocarcinoma y adenoacantoma o carcinoma escamoso. Puede ser difuso o circunscrito y a veces toma la forma polipóide. Se han observado casos en que se desarrolla sobre un pólipo endometrial. Sus características microscópicas en general corresponden a las encontradas en las lesiones de naturaleza maligna o sean anaplasia y metaplasia celular, cambios de afinidad tintorial, pérdida de la polaridad de las células, polimorfismo celular, invasión de los estratos vecinos, etc. En la hiperplasia endometrial la imagen histológica corresponde a la de "queso suizo" (término usado por Novak) con aumento de los elementos tisulares tanto epiteliales como del estroma, el epitelio es más alto que el normal, las glándulas profundas, hiperplásicas e irregulares, a veces quísticas. Las células muestran mitosis frecuentes y no se encuentran modificaciones del período secretor (vacuolas, glicógeno, etc.). Frecuentemente se observan zonas infiltrativas con cambios degenerativos de origen no infeccioso.

El aumento del tamaño del útero que acompaña generalmente al carcinoma del endometrio hace pensar que los factores desencadenantes de la anarquia celular de tipo maligno también influven sobre el tejido muscular y conjuntivo.

Se ha prescindido de la clasificacion clínica del carcinoma según su localización o invasión ya sea el endomerrio o a las demás capas de tejido de la matriz (miometrio y serosa). Actualmente la clasificación clínica se reduce a ures estados: Primer estado: clinicamente operable. Segundo estado: técnicamente operable aunque estén invadidos órganos vecinos y Tercer estado: no coperable.

El carcinoma del útero puede permanecer por largo tiempo localizado al indometrio. Posteriormente se propaga al miometrio y puede producir invasión por contiguidad a las trompas y ovarios. peritoneo abdominal, canal del cervix o ragina, aunque siendo rara esta última y la invasión a la trompa. Las metástasis por vía linfática o sanguínea se hacen al grupo de ganglios situados en las cadenas iliacas y para-aórticas inferiores o trompa y orario. A distancia se hacen a columna vertebral y pulmón.

1-Importancia del legrado-biópsia en el diagnostico de las hemorragias uterinas.

Si bien es cierto que las hemorragias de tipo funcional son numerosas, exisun gran variedad de lesiones orgánicas endometriales que originan este síntoma y el único medio diagnóstico de ellas es el legrado-biópsia. No es necesario insistir sobre lo dicho respecto a las lesiones malignas, pero se debe recordar que los pólipos endometriales, restos ovulares, las endometritis crónicas, la mola hidatidiforme, los endometrios atípicos, la adenomiosis, etc., producen hemorragia y generalmente no es posible llegar a su diagnéstico sino mediante el legrado. Si stiene en cuenta que la intervención es en muchos de estos casos a la vez indi. ción terapéutica hay un motivo más para insistir en su conveniencia. Clínicac..mente se pueden descartar algunas lesiones aparentes causantes de hemorragia, intre ellas: pólipos del endocervix en especial cuando están prolapsados, fibro. miomas uterinos, tumores del ovario, enfermedades generales vervigracia: diáte- 
sis hemorrágica, lesiones cardíacas y renales, hemorragias del embarazo. Sin em bargo en algunas de éllas, en especial en la fibromatosis uterina es convenient y a veces necesario descartar una entidad maligna asociada.

III.-Condiciones y técnica del legrado. Exámenes previos. Anestesia, Morb? lidad. Mortalidad.

En el Instituto Nacional de Cancerología se procede con las pacientes qu consultan por el sintoma hemorragia genital en la siguiente forma. Se descar tan todas aquellas lesiones aparentes que la puedan provocar. en especial la de cuello, vulva y vagina, también las relacionadas con el embarazo y las enfer medades orgánicas de tipo general. Posteriormente según el criterio del gine cólogo se indica el legrado-biópsia de endometrio y de endocérvix en muestra apartes para su estudio anatomo-patológico. Gracias a este procedimiento se har confirmado multitud de casos sospechosos de carcinoma de endometrio y se har descubierto casos insospechados, como también se logra una orientación diag nóstica sobre lesiones benignas que el examen clínico no lo habría permitido. S la lesión no es maligna la paciente se envía a otros servicios hospitalarios o a si médico particular y en caso de que resulte maligna se ordena el tratamiento in dicado. Además se instruye a las enfermas sobre la necesidad de acudir a la con sulta si presenta nueva sintomatología genital.

De rutina se ordenan los siguientes exámenes: clinico general, cuadro he mático, N.P.N. glicemia, parcial de orina, para autorizar la anestesia general Algunas veces, según las condiciones de la enferma se practica anestesia local El anestésico más utilizado es el penthotal por vía renosa. previa medicación La intervecnión es la misma que para cualquier legrado uterino y con cierta prác tica apenas se lleva unos pocos minutos. Dicho legrado debe ser completo. E accidente más frecuente es la hemorragia posterior facilmente controlable. Po esta razón las enfermas permanecen hospitalizadas por $2+$ horas. Se han presen tado algunos casos de perforación uterina, especialmente en úteros reblandecido: por lesiones carcinomatosas, pero con reposo y antibioticos esta complicaciór no es grave. La mortalidad en los casos estudiados es nula.

IV.-Cuadros estadísticos.

Se presentan a continuación los resultados obtenidos en los 100 casos estu diados en serie desde enero de 1952 a febrero de 1953.

I.-Incidencia de hemorragia uterina patológica y de carcinoma primitiv de endometrio por grupos de edades:

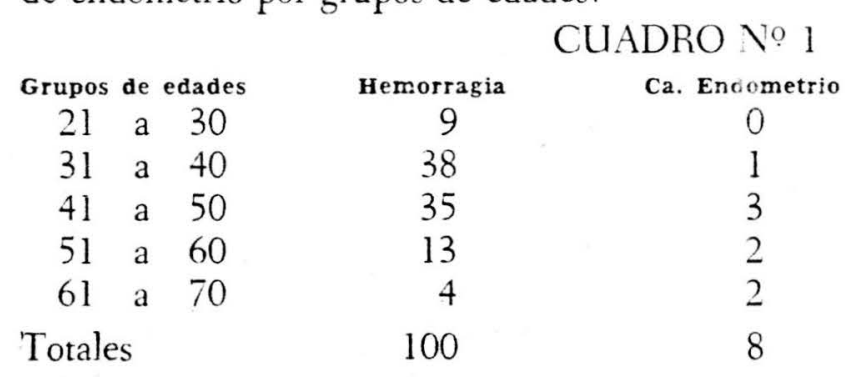

Porcentajes Malignidac

8,57

15,39

50 


\section{Observaciones:}

a) Se prescinde en este cuadro de un caso de carcinoma de endometrio por propagación de epi del cuello y un caso de mola degenerada.

b) La mayor incidencia de hemorragia corresponde a la tercera y cuarta décadas.

c) El porcentaje sobre el total de los casos de Ca. de endometrio es de $8 \%$ $y$ de malignidad en total de $10 \%$.

d) Es importante anotar que el porcentaje de $\mathrm{Ca}$. de endometrio se hace muy alto en la quinta décina y es altísimo en la sexta durante la cual en los casos estudiados llega al $50 \%$.

\section{CUADRO No 2}

Resultado anatomo-patológico en los 100 casos, por orden de frecuencia:

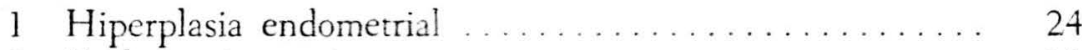

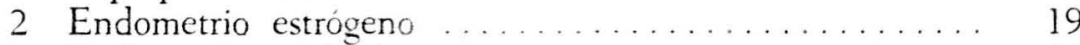

3 Endometrio atrófico ..................... 11

4 Endometrio secretor o pre-g. ................ 10

5 Carcinoma de endometrio ................... 9

6 Restos ovulares .......................... 6

7 Endometrio atípico .................... 5

8 Endometritis sub-aguda $\ldots \ldots \ldots \ldots \ldots \ldots \ldots \ldots \ldots \ldots$

9 Pólipo endometrial ...................... 2

10 Endometrio tipo mixto .................. 1

11 Adenomiosis ............................ 1

12 Células deciduales malignas $\ldots \ldots \ldots \ldots \ldots \ldots \ldots \ldots$

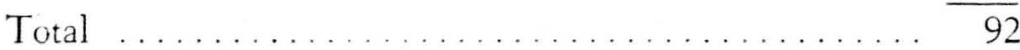

Muestra insuficiente ...............

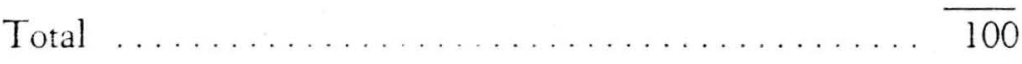

\section{Observaciones:}

a) Los casos de muestra insuficiente se han seguido posteriormente sin repetir legrado por no haber presentado sintomatología. En los casos de endometrio atípico se ha seguido conducta semejante.

b) En los diagnósticos como endometrio estrógeno no se aclaró la fase del cido en que fueron tomados; queda la duda de si se trata de endometrio estrógeno persistente a pesar de que se ordene el legrado en la segunda fase del ciclo cuando ésta se conoce. 


\section{CUADRO No 3}

Otras lesiones ginecológicas diagnosticadas clínicamente en los casos est diados, por orden de frecuencia:

1 Cervicitis crónica ... . . . . . . . . . . . . . . . . . 29

2 Fibromatosis uterina .................... 14

3 Pólipos endocervicales .................... 8

4 Salpingitis crónica ...................... 3

5 Parametritis crónica ...................... 3

6 Varicocele pelviano ..................... 1

7 Prolapso genital ....................... 1

\section{Observaciones:}

a) Es notable el alto porcentaje de cervicitis. Se pone de presente en est: observaciones la fibromatosis uterina y los pólipos endocervicales como causas c hemorragias.

b) Desde luego este cuadro no tiene ninguna significación estadístic puesto que en una enferma han podido coincidir varias de estas lesiones.

\section{CUADRO NO 4}

Otras lesiones malignas asociadas en las 100 enfermas:

1 Epi. del cuello, Estado III

2 Carcinoma del ovario metastásico

3 Carcinoma del ovario primitivo

\section{CUADRO No 5}

La menopausia en relación con algunas de las entidades encontradas:

1 En el total de los casos (100) ........... 29

Porcentaje:

2 En $9 \mathrm{Ca}$. de endometrio $29.00 \%$

3 En 24 hiperplasias 6. $66,66 \%$

4 En 11 endometrios atróficos ........... 11

\section{Observaciones:}

a Este cuadro confirma que el $\mathrm{Ca}$. del endometrio se presenta con may frecuencia en pacientes menopáusicas y por lo tanto en ellas la hemorragia ut rina debe inclinar al médico a hacer una investigación más cuidadosa de causa.

b) En las hiperplasias por el contrario la incidencia durante la menopa sia es mucho menor. En estas observaciones apenas del $20 \%$.

c) En endometrio atrófico es propio generalmente de enfermas menopáu cas, por la involución genital de esta edad. En estas observaciones es del 100 


\begin{tabular}{|c|c|c|c|c|c|c|c|c|}
\hline Historia & Edad & $\begin{array}{l}\text { Nimero de } \\
\text { Embarazo }\end{array}$ & $\begin{array}{l}\text { Jlimo } \\
\text { Parto }\end{array}$ & $\begin{array}{l}\text { Tiempo de } \\
\text { Menopausia }\end{array}$ & $\begin{array}{l}\text { Lapso sin- } \\
\text { tomatologia } \\
\text { 1a Consul. }^{\text {a Cons }}\end{array}$ & Sintomatologia & $\begin{array}{l}\text { Tratamiento Tipo } \\
\text { Histol. }\end{array}$ & Observaciones \\
\hline 28.166 & 28 & 3 & 2 & No & 4 & Metrorragia. & Legrado Mola Deg. & En observación. \\
\hline 30.281 & 30 & 6 & 4 & No & 7 & Metrorragia. & $\begin{array}{l}\text { Radium RX. Ca. } \\
\text { Escano-cal. }\end{array}$ & Con Epi de cuello. \\
\hline 20.155 & 38 & 6 & 5 & No & 8 & Metrorragia. & Ninguno Adenoc. & Perdida. \\
\hline 29.831 & 43 & 5 & - & 2 & 3 & $\begin{array}{l}\text { Flujo fétido. He- } \\
\text { morragia. }\end{array}$ & $\begin{array}{l}\text { Radium Intra-ut. } \\
\text { Adenorcarc. }\end{array}$ & $\begin{array}{l}\text { Tratamiento incompleto } \\
\text { por desaparieión. }\end{array}$ \\
\hline 30.028 & 45 & 0 & 13 & No & 12 & Metrorragia. & $\begin{array}{l}\text { Radium Histerectomía } \\
\mathbf{R} \times \text { Adenoc. }\end{array}$ & $\begin{array}{l}\text { Ca. Metastásico ovario. } \\
\text { Rayoos X. Incoinpletos. }\end{array}$ \\
\hline 29.654 & 48 & 2 & $m$ & 10 & 12 & $\begin{array}{l}\text { Leucorrea. Hemo- } \\
\text { rragia un dia. }\end{array}$ & $\begin{array}{l}\text { Histerectomia Rayos } \mathrm{X} \\
\text { Adenocare. }\end{array}$ & Tratamiento completo. \\
\hline 29.907 & 55 & 0 & 23 & 8 & 5 & Metrorragia. & $\begin{array}{l}\text { Radium Histerectomla. } \\
\text { Ca. Escamoe!. }\end{array}$ & $\begin{array}{l}\text { Tratamiento } 8 \text { meses } \\
\text { más tarde por desapa- } \\
\text { rición. }\end{array}$ \\
\hline 29.779 & 55 & 0 & - & 12 & 12 & $\begin{array}{l}\text { Flujo fétido he- } \\
\text { morrágico. }\end{array}$ & $\begin{array}{l}\text { Radium Adenocarcino- } \\
\text { ma. }\end{array}$ & $\begin{array}{l}\text { Tratamiento completo } \\
\text { paliativo. }\end{array}$ \\
\hline 29.486 & 62 & 1 & 19 & 12 & 2 & $\begin{array}{l}\text { Metrorragia. } \\
\text { Abombamiento } \\
\text { abdominal. }\end{array}$ & $\begin{array}{l}\text { Histerectomía Adeno- } \\
\text { carcinoma. }\end{array}$ & $\begin{array}{l}\text { Con Ca. primitivo del } \\
\text { ovario. Tratamiento in } \\
\text { completo. }\end{array}$ \\
\hline 28.116 & 70 & 9 & 35 & 30 & 8 & Metrorragia. & Radium Adenoc. & Tratamiento com \\
\hline
\end{tabular}


C. G. $8558 \quad 46 \quad 1 \frac{11}{2}$ meses Metrorragia. Dolor hipogástrico. 7 partos.

$8075 \quad 45 \quad 16 \quad$ meses

I. de J. 7378

6 años

Metrorragia abundante. 6 partos y 3 abortos. Prolapso, Hernia umbilical. Utero grande 9 ctms de histerometría. Dismenorrea. 1. H. $7007 \quad 48 \quad 6$ meses 5 partos, 1 aborto. Menopausia
hace 3 años. Utero grande. 9 te ovárico. Dismenorrea.

$\begin{array}{llll}5586 & 40 & 1 & \text { año }\end{array}$

Dolor hipogástrico. Metrorragia. Utero grande.

$5487 \quad 36 \quad 5 \quad$ años

Metrorragia. Utero normal. Metropatia hemorrágica antigua.

$\begin{array}{llll}3606 & 37 & 1 & \text { año }\end{array}$

$2720 \quad 36$

$2507 \quad 39$

$2506 \quad 41$

6

meses

5039

Esteril. Dismenorrea. Dolor hipogástrico. Metrorragia.

Dolor hipogástrico. Metrorra- Histerectomía subtotal. gia. Utero grande.

Metrorragia. Prurito. Masa ute- Histerectomía subtotal. rina del tamaño de cabeza fetal. Dolor.

Hipermenorrea, 13 partos. Metrorragia. Utero grande 10 ctms. de histerometría.

T. $R$. $54 \quad 1$ aก̃o
Lesión del ombligo.

Metrorragia. Anemia. $8 \mathrm{ctms}$, de histerometría.
Histerectomia total anexos.

Histerectomía total.

Histerectomía total.

Histerectomia total conservando anexo izquierdo.

Histerectomía total.

Histerectomia total conservando un anexo.

Histerectomía subtotal.

Histerectomía subtotal.

Extirpación.

Histerectomía total.
Hiperplasia endometrial. Adenomiosis Fibrosis ovariana.

Fase estrogénica vanzada. Adenomiosis e hiperplasia del endometrio.

Miomas. Endometriosis de ovario y adenomiosis.

Fndometriosis uterina $y$ teratoma ovariano.

Endometritis. Atrofia endometrial y Ademiosis.

Endometriosis del ovario. Hiperplasia del endometrio.

Fibromioma calcificado $\mathrm{y}$ adenomiosis.

Fibromioma. Endometriosis del ovario y embarazo ectópico.

Hiperplasia del endometrio. Adenomiosis.

Adenomiosis. (Glándulas fase secretoria).

Endometriosis.

Adenomiosis. 

M. M.
2944

M. M.

40

3 años

A. G. $\quad 15766 \quad 32$

6

meses

Tumor parauterino izquierdo. Edema miembro inferior izquierdo.

Extirpación del tumor sin clivaje.

Dismenorrea. Fornix posterior invadido. Metrorragia. Utero normal. Douglas invadido.

Histerectomía total.

Dolor hipogástrico. Metrorragia. Extirpación. Flujo. Pólipo. Utero grande. Dismenorrea.

B. C. $15433 \quad 30 \quad 4$ meses Pólipo exocervical.
Dolor lumbar izquierdo. Disuria Dismenorrea.
A. M. $\quad 15409 \quad 40 \quad 1 \quad$ mes

J. L. $\quad 14999 \quad 36 \quad 1 \quad$ año

M. B. $\quad 14606 \quad 46$

5 meses

Metrorragia a $\operatorname{los} 8$ años de la menopausia.

Metrorragia. Anemia.

A. J. $\quad 14160 \quad 40 \quad 15$ años Polihipermenorrea indolora

I. P. $\quad 12578 \quad 38$

2 años

Dolor hipogástrico. Metrorragia. $91 / 2$ ctms. de histerometría. Dismenorrea.

S. M. $12210 \quad 42 \quad 6$ meses Metrorragias. Utero grande. Cuadro apendicular agudo.

L. E. $\quad 12151 \quad 46 \quad 8$ meses Metrorragias. Dolor hipogástrico con irradiación lumbar.

P. A. $11601 \quad 4520$ años Prolapso grado 3. Utero grande.
Extirpación.

Histerectomía subtotal sin anexos.

Histerectomía completa con anexos.

Histerectomía subtotal sin anexos.

Histerectomía subtotal sin anexos.

Histerectomía subtotal sin anexos.

Histerectomía subtotal sin anexos.

Histerectomín total sin anexos.

Spallding-Richardson.
Endometriosis de la base del ligamento ancho.

Endometriosis interna y externa pélvica.

Mioma submucoso con adenomiosis.

Mioma submucoso con adenomiosis.

Leiomioma uterino y adenomiosis.

Pólipo endometrial y ademiosis.

Hiperplasia glándulo-quística del endometrio y adenomiosis.

Hiperplasia glánduloquística del endometrio. Adenomiosis.

Adenomiosis y pequeño Leiomioma .

Adenomiosis y Endometriosis.

Adenomiosis. Epitelio normal (fase estrogénica).

Adenomiosos. Hiperplasia glándulo-quística del endometrio. 


\begin{tabular}{|c|c|c|c|c|c|c|c|}
\hline Nombre & Biópsia & Edad & & oluclón & Datos Clínicos & Intervención & Patología \\
\hline E. C. & 11380 & 36 & 4 & meses & $\begin{array}{l}\text { Tumor inguinal que después de } \\
\text { la menstruación entra en re- } \\
\text { ceso. Fístula hace } 3 \text { meses que } \\
\text { sangró en el periodo mens- } \\
\text { trual. }\end{array}$ & $\begin{array}{l}\text { Extirpación e histerecto- } \\
\text { mia total con anexos. }\end{array}$ & Endometriosis cutánea. \\
\hline R. M. & 10102 & 45 & 3 & años & $\begin{array}{l}\text { Metrorragias y dolor. Utero } \\
\text { grande. } 9 \text { ctms. de histerome- } \\
\text { tria. Dismenorrea. }\end{array}$ & Histerectomía total. & Adenomioma circunscrito. \\
\hline L. C. & 9842 & 35 & 6 & meses & $\begin{array}{l}\text { Dolor. Utero grande. Dismeno- } \\
\text { rrea. } 13 \text { ctms. de histerome- } \\
\text { tría. }\end{array}$ & Histerectomía subtotal. & $\begin{array}{l}\text { Fibromiomas, Endometrio- } \\
\text { sis. }\end{array}$ \\
\hline
\end{tabular}




\section{SUMARIO}

Se estudian 27 casos de endometriosis, presentados en Medellín, en el lapso de 6 años y 8 meses, de noviembre 25 de 1946 a julio 15 de 1953 , con el resumen de sus historias clínicas e informes de laboratorio de Anatomía Patológica.

En el mismo tiempo anotado, se operaron en el Servicio de Ginecología 17 casos de quistes hemáticos de ovario, pero el estudio histológico fue negativo para esta entidad.

La edad promedio de las pacientes fue de 38.7 años. La endometriosis interna es, en que nuestra serie, cuatro veces mayor que la externa, lo cual anda cn desacuerdo con las estadísticas universales. Esto merece estudios ulteriores de comprobación.

Los trastornos hemorrágicos catameniales son el síntoma predominante con $77.7 \%$. En seguida la dismenorrea con el $59.2 \%$. Por último el dolor hipogástrico con el $40.7 \%$.

Dos entidades patológicas se encontraron asociadas a la endometriosis en las dos terceras partes de los casos: la hiperplasia glándulo-quística en el $29.8 \%$ y los fibromiomas en el $33.3 \%$. La entidad típica sin asociación sólo se halló en el $22.2 \%$.

\section{B I B L I O G R A F I A :}

Meigs and Sturgis: "Progress in Gynecology", Vol. 11. - 1950.

Novak Emil: "Pelvic Endometriosis". A. J. Obst. and Gyn., 22. - 1931.

Eben T. Bennet: A. J. Obst and Gyn. Vol. 65, No 1. 100. - 1953.

Javert C. T.: A. J. Obst. and Gyn. Vol. 62. 477. - 1951.

Te Linde and Scott R. B.: A. J. Obst. and Gyn. Vol. 60. 1.147. - 1950. 